\title{
Risk stratification of castration-resistant prostate cancer patients treated with cabazitaxel
}

\author{
TAKEO KOSAKA, HIROSHI HONGO, RYUICHI MIZUNO and MOTOTSUGU OYA \\ Department of Urology, Keio University School of Medicine, Tokyo 160-8582, Japan
}

Received March 22, 2018; Accepted September 3, 2018

DOI: $10.3892 / \mathrm{mco} .2018 .1724$

\begin{abstract}
Patient characteristics before administering the first cycle of cabazitaxel for metastatic castration-resistant prostate cancer (mCRPC) were collected to assess prognostic factors for overall survival (OS). Multivariate analysis revealed that prostate-specific antigen $(\mathrm{PSA}) \geq 100 \mathrm{ng} / \mathrm{ml}$ prior to cabazitaxel treatment, visceral metastasis, and low absolute monocyte count were independent prognostic indicators for OS. The aim of the present study was to investigate prognostic biomarkers in patients treated with cabazitaxel among Japanese metastatic castration-resistant prostate cancer (mCRPC) patients. In this retrospective study, 45 patients with $\mathrm{mCRPC}$ treated with cabazitaxel were reviewed retrospectively. Clinicopathological factors and laboratory data before administering the first cycle of cabazitaxel were collected to assess the prognostic factors for overall survival (OS). Treatment was generally well tolerated, with a median of 5 cycles (range, 1-26). Median OS from the start of cabazitaxel treatment was 16.1 months (95\% confidence interval 6.8-25.5). Univariate analysis revealed that poor performance status, visceral metastasis, hemoglobin $<11 \mathrm{mg} / \mathrm{dl}$, absolute monocyte count $<400 / \mu 1$, and prostate-specific antigen $\geq 100 \mathrm{ng} / \mathrm{ml}$ prior to cabazitaxel treatment $(\mathrm{P}=0.002)$ were significantly associated with shorter OS. Multivariate analysis revealed that PSA $\geq 100 \mathrm{ng} / \mathrm{ml}$ prior to cabazitaxel treatment, visceral metastasis, and absolute monocyte count $<400 / \mu 1$ were independent prognostic indicators for OS. Based on the relative risk of death, patients with mCRPC before cabazitaxel therapy were divided into three risk groups: Low, intermediate, and high $(\mathrm{P}<0.001)$. In conclusion, the practical implications of our results may assist in tailoring the introduction of cabazitaxel.
\end{abstract}

Correspondence to: Dr Takeo Kosaka, Department of Urology, Keio University School of Medicine, 35 Shinanomachi, Shinjuku-ku, Tokyo 160-8582, Japan

E-mail: takemduro@gmail.com, takemduro@keio.jp

Key words: castration-resistant prostate cancer, chemotherapy, cabazitaxel, overall survival, risk stratification

\section{Introduction}

Prostate cancer is the most frequently diagnosed cancer and the second leading cause of cancer-related death in the United States (1-4). In Japan, prostate cancer is estimated to be the most frequently diagnosed cancer and sixth highest cause of cancer-related death in the male population (5).

Cabazitaxel is a next-generation taxane that is indicated for the treatment of patients with metastatic castrationresistant prostate cancer (mCRPC) previously treated with a docetaxel-containing regimen (6-8). The phase III TROPIC study revealed that cabazitaxel provided an overall survival (OS) benefit in patients with mCRPC progressing during or after docetaxel. In a phase I study of cabazitaxel in Japanese patients with $\mathrm{mCRPC}$, the safety and antitumor activity based on prostate-specific antigen (PSA) response rate in Japanese patients were found to be similar to the results of previous studies in Caucasian patients. Cabazitaxel was approved worldwide for the treatment of patients with MCRPC previously treated with a docetaxel-containing regimen (9-12). However, the prognostic or predictive biomarker regarding OS have not yet been fully characterized.

The aim of the present study was to investigate prognostic biomarkers in patients treated with cabazitaxel in Japanese mCRPC patients.

\section{Patients and methods}

Patients. In this retrospective observational study, 45 patients with mCRPC treated with cabazitaxel at Keio University Hospital from 2014 to 2017 were identified. All patients were histologically confirmed as having adenocarcinoma of the prostate with clinical or radiologic evidence of metastatic disease and had disease progression during treatment consisting of complete androgen blockade hormone therapy and docetaxel. All patients received cabazitaxel at $20-25 \mathrm{mg} / \mathrm{m}^{2}$ administered intravenously every 3-4 weeks, together with prednisone $5 \mathrm{mg}$ twice daily. Prophylactic administration of granulocyte-colony stimulating factor was prescribed to all patients.

For this study, clinico-pathologic and laboratory data for each patient were obtained retrospectively from medical records. These data were collected to examine the clinical association with progression-free survival (PFS) and OS. PFS was defined as an increase in PSA values $\geq 25 \%$ relative to the pretreatment PSA value or radiologic progression 
according to the Response Evaluation Criteria In Solid Tumors (RECIST) guidelines. OS was calculated from the date of the start of cabazitaxel treatment to the date of death or date of last follow-up. Adverse events were classified in accordance with the Common Terminology Criteria for Adverse Events (CTCAE) dictionary version 4.0.

Our study was designed as a retrospective analysis, and approval was obtained from the Institutional Review Board of our institution.

Statistical analysis. The continuous variables and categorical variables of different groups were compared using the chi-square test and Mann-Whitney U test, respectively. The Kaplan-Meier method was used to estimate the event-time distributions for PFS and OS, and the log-rank test was then used to assess significance. Univariate Cox regression models were used to adjust for potential confounders in predicting OS. Covariates with significant P-values $(<0.05)$ in univariate analysis were included in the multivariable analysis. Categorized variables were assessed in multivariate models using Cox proportional hazard regression models with a stepwise forward selection method. For all statistical analyses, tests were two-sided and $\mathrm{P}<0.05$ was considered to indicate statistical significance. All statistical analyses were performed using the Statistical Package of the Social Sciences, version 24.0 (SPSS, Chicago, IL, USA).

\section{Results}

Patient characteristics. A summary of the characteristics of the 45 mCRPC patients in this study is shown in Table I. Median age was 71 years. The Eastern Cooperative Oncology Group performance status (ECOG PS) score was 0 and $1 / 2$ in 87.8 and $12.2 \%$ of patients, respectively. The median baseline PSA level was $124.3 \mathrm{ng} / \mathrm{ml}$ (range, 0.17-11,660). Major sites of disease included bone $(97.8 \%)$. The median number of prior docetaxel cycles was 8 (range, 3-43). Cabazitaxel was applied as the second- or third-line treatment in $11(7.3 \%)$ patients and as fourth-line treatment or more in $34(75.6 \%)$ patients. Treatment was generally well tolerated, with a median of 5 cycles (range, 1-26).

Univariate and multivariate analysis of overall survival. Median OS from the start of cabazitaxel treatment was 16.1 months [95\% confidence interval (CI) 6.8-25.5] (Fig. 1). PSA decline of $>50$ or $>30 \%$ was observed in $13(26.1 \%)$ and $16(34.8 \%)$ patients, respectively. Our primary objective was to examine whether the laboratory data or clinic-pathologic indicators before administering the first cycle of cabazitaxel had any associations between OS in men with $\mathrm{mCRPC}$ receiving cabazitaxel.

To identify the clinical-biologic parameters associated with OS in patients treated with cabazitaxel chemotherapy, univariate and multivariate analyses were performed using a Cox proportional hazard regression model.

Univariate analysis revealed that poor PS $(\mathrm{P}<0.001)$, visceral metastasis $(\mathrm{P}=0.036)$, hemoglobin $<11 \mathrm{mg} / \mathrm{dl}$ $(\mathrm{P}<0.001)$, absolute monocyte count $<400 / \mu 1(\mathrm{P}=0.026)$, PSA $\geq 100 \mathrm{ng} / \mathrm{ml}$ prior to cabazitaxel treatment $(\mathrm{P}=0.002)$ were significantly associated with shorter OS (Table II).
Table I. Characteristics of patients treated with cabazitaxel.

\begin{tabular}{lc}
\hline Characteristic & Value $(\mathrm{N}=45)$ \\
\hline Age, years, median (range) & $71(46-85)$ \\
Age group, $\mathrm{n}(\%)$ & \\
$<75$ years & $37(85.4)$ \\
75 -years & $8(14.6)$ \\
ECOG PS, $\mathrm{n}(\%)$ & $38(87.8)$ \\
0 & $7(12.2)$ \\
1,2 & $124.3(0.17-11,660)$ \\
PSA at baseline, ng/ml, median (range) & \\
Sites involved, $\mathrm{n}(\%)$ & $44(97.8)$ \\
Bone & $17(38.6)$ \\
Lymph nodes & $13(9.1)$ \\
Visceral metastasis & $10(22.7)$ \\
Prior surgery, $\mathrm{n}(\%)$ & $20(43.2)$ \\
Prior radiotherapy, $\mathrm{n}(\%)$ & \\
Prior 2nd AR targeting lines, $\mathrm{n}(\%)$ & $29(60.9) / 22(48.9)$ \\
ENZA/ABI & \\
Prior docetaxel-containing regimens, & \\
$\mathrm{n}(\%)$ & $11(7.3)$ \\
1,2 & $17(37.8)$ \\
3 & $17(37.8)$ \\
4 or more & $8(3-43)$ \\
Total prior docetaxel cycle, median \\
(range)
\end{tabular}

ECOG PS, Eastern Cooperative Oncology Group performance status; PSA, prostate-specific antigen; ENZA, Enzalutamide; ABI, abiraterone; $\mathrm{Hb}$, hemoglobin; ALP, al; AMC, absolute monocyte count; NLR, Neutrophil to Lynphocyte ratio.

Multivariate analysis revealed that PSA $\geq 100 \mathrm{ng} / \mathrm{ml}$ prior to cabazitaxel treatment [hazard ratio $(\mathrm{HR})=4.375 ; 95 \%$ CI 1.755-10.91, $\mathrm{P}=0.002]$, visceral metastasis $(\mathrm{HR}=2.926$; 95\% CI 1.196-7.157, $\mathrm{P}=0.019)$, and absolute monocyte count $<400 / \mu 1(\mathrm{HR}=2.955 ; 95 \%$ CI 1.219-7.165, $\mathrm{P}=0.026)$ were independent prognostic indicators for OS.

Risk stratification according to three variables: PSA, visceral metastasis, and absolute monocyte count. We distributed the patients into three different groups according to PSA, visceral metastasis, and absolute monocyte count, which were the three statistically significant variables found by the multivariate Cox regression analysis (Fig. 2). The relative risk of death was calculated using the formula, $\exp (4.375 \times$ PSA $+2.955 \times$ monocyte count $+2.926 \mathrm{x}$ visceral metastasis) for OS. In this equation the PSA equaled 1 if the PSA level was $>100 \mathrm{ng} / \mathrm{ml}$, and it equaled 0 if the PSA level was $\leq 100 \mathrm{ng} / \mathrm{ml}$. Absolute mono- 
A

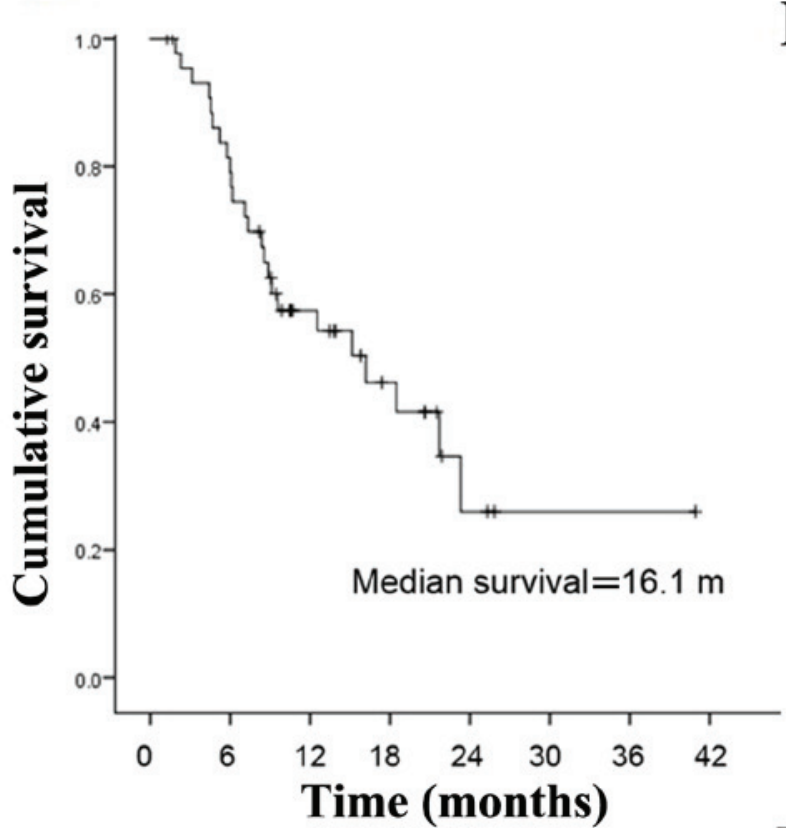

$\mathbf{C}$

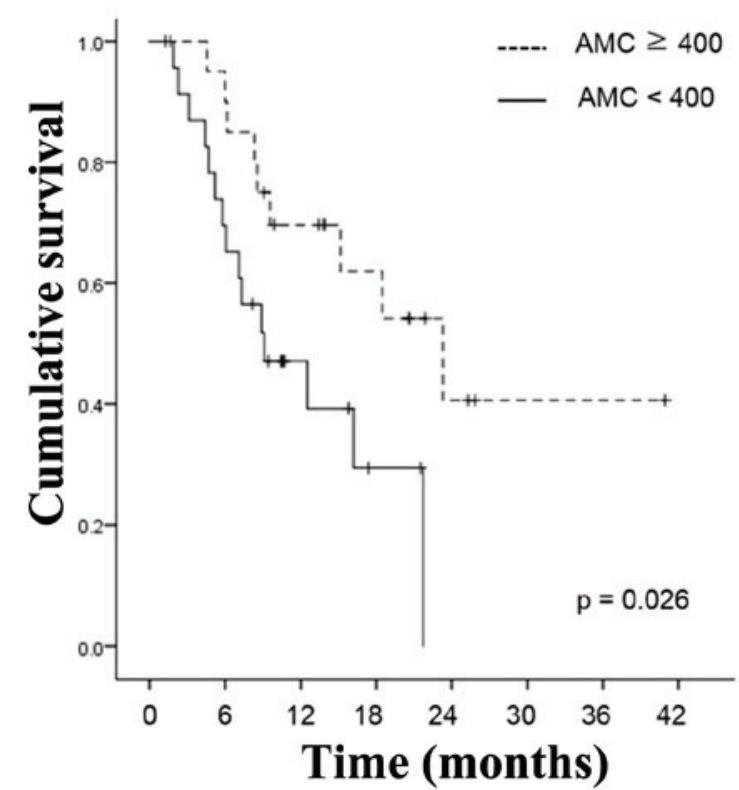

B

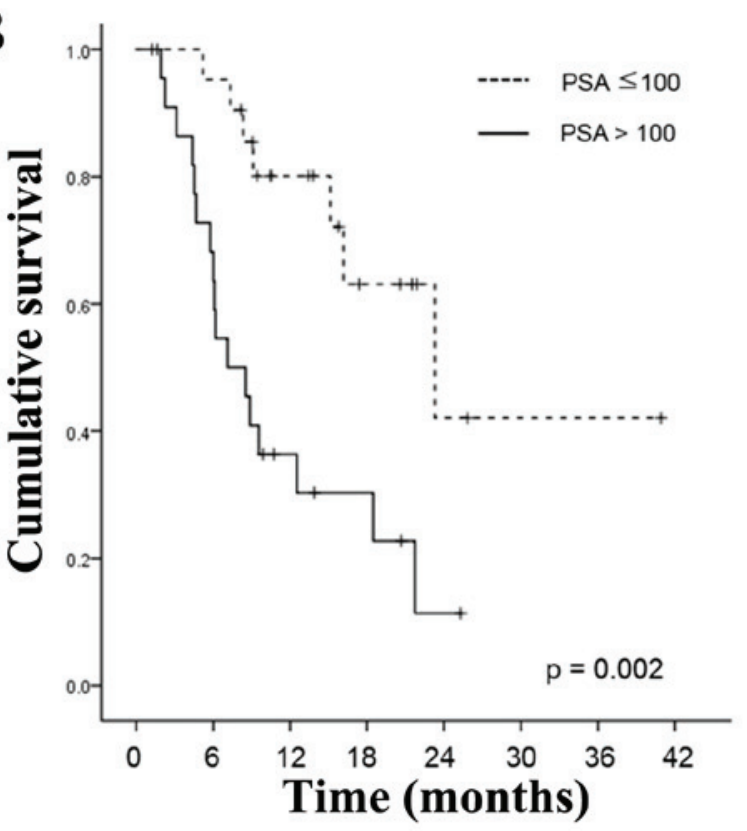

D

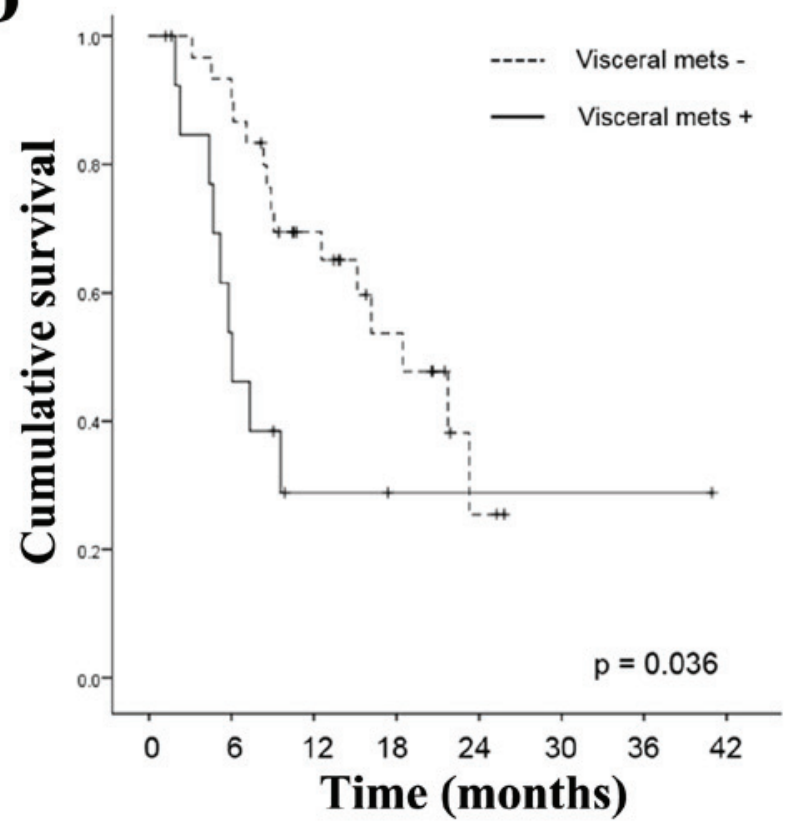

Figure 1. (A) Kaplan-Meier curve for time to OS in the total population (n=45). (B) Kaplan-Meier curve for time to OS in the PSA specified population (n=45). (C) Kaplan-Meier curve for time to OS in the absolute monocyte count specified population (n=45). (D) Kaplan-Meier curve for time to OS in the visceral metastasis specified population $(n=45)$.

cyte count equaled 1 if the count was $<400 / \mu 1$ and 0 if it was $\geq 400 / \mu 1$. Visceral metastasis equaled 1 if visceral metastasis was present and 0 if there was no metastasis. Based on the relative risk of death, patients with mCRPC before cabazitaxel therapy were divided into three risk groups: Low ( 0 factors, relative risk=1), intermediate ( 1 or 2 factors; relative risk=2.926-7.33 for OS), and high (3 factors; relative risk=10.26 for OS). According to the risk stratification based on prognostic factors, 9 patients $(20.0 \%)$ were in the low-risk group, 5 patients $(11.1 \%)$ were in the high-risk group, and 31 patients $(68.9 \%)$ were in the intermediate-risk group. Median OS from the start of cabazitaxel treatment was 23.3 months in the low-risk group, 16.1 months in the intermediate-risk group, and 2.5 months in the high-risk group. The differences among the groups were significant $(\mathrm{P}<0.001)$.

\section{Discussion}

A phase I cabazitaxel study in Japan did not demonstrate efficacy, and the efficacy and prognostic indicators for cabazitaxel in Japan are still unclear, although cabazitaxel was the first agent demonstrating a survival benefit in men with mCRPC progressing during or after docetaxel $(6,13,14)$.

In the PSA era, PSA has played a crucial role in prostate cancer diagnosis, the definition of castration-resistant prostate cancer (CRPC), and the introduction of cabazitaxel indicating the extent of disease progression. It has been 
Table II. Results of unvariate and multivariate analysis influencing OS.

\begin{tabular}{|c|c|c|c|c|c|}
\hline \multirow[b]{2}{*}{ Variables } & \multirow[b]{2}{*}{ No. $(\%)$} & \multirow{2}{*}{$\begin{array}{c}\begin{array}{c}\text { Univariate } \\
\text { analysis }\end{array} \\
\text { P-value }\end{array}$} & \multicolumn{3}{|c|}{ Multivariate analysis } \\
\hline & & & HR & $95 \% \mathrm{CI}$ & P-value \\
\hline \multicolumn{6}{|l|}{ Age, years } \\
\hline$\leq 75$ & $9(20)$ & 0.808 & & & \\
\hline$>75$ & $36(80)$ & & & & \\
\hline \multicolumn{6}{|l|}{ PS } \\
\hline 1,2 & 7 (15.6) & $<0.001$ & & & \\
\hline 0 & $38(84.4)$ & & & & \\
\hline \multicolumn{6}{|c|}{ Visceral metastasis } \\
\hline Yes & $13(28.9)$ & 0.036 & 2.926 & $1.196-7.157$ & 0.019 \\
\hline No & $32(71.1)$ & & & & \\
\hline \multicolumn{6}{|c|}{ PSA (ng/ml) } \\
\hline$>100$ & $23(51.1)$ & 0.002 & 4.375 & $1.755-10.91$ & 0.002 \\
\hline$\leq 100$ & $22(48.9)$ & & & & \\
\hline \multicolumn{6}{|c|}{$\mathrm{Hb}(\mathrm{mg} / \mathrm{dl})$} \\
\hline$\leq 11$ & $18(40)$ & $<0.001$ & & & \\
\hline$>11$ & $27(60)$ & & & & \\
\hline \multicolumn{6}{|l|}{$\operatorname{AMC}(/ \mu 1)$} \\
\hline$<400$ & $25(55.6)$ & 0.026 & 2.955 & $1.219-7.165$ & 0.017 \\
\hline$\geq 400$ & $20(44.4)$ & & & & \\
\hline \multicolumn{6}{|l|}{ NLR } \\
\hline$\geq 4.3$ & $23(51.1)$ & 0.097 & & & \\
\hline$<4.3$ & $22(48.9)$ & & & & \\
\hline \multicolumn{6}{|l|}{$\operatorname{ALP}(\mathrm{IU} / \mathrm{l})$} \\
\hline$>350$ & $17(37.8)$ & 0.268 & & & \\
\hline$\leq 350$ & $28(62.2)$ & & & & \\
\hline
\end{tabular}

ECOG PS, Eastern Cooperative Oncology Group ECOG performance status; PSA, prostate-specific antigen; Hb, hemoglobin; AMC, absolute monocyte count; NLR, Neutrophil to Lynphocyte ratio; ALP, alkaline Phosphatase.

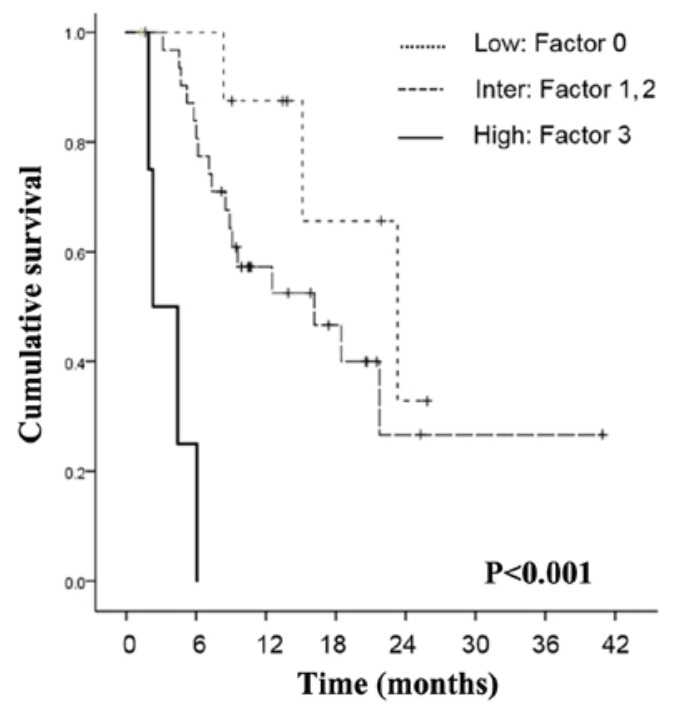

Figure 2. Kaplan-Meier curves of OS of patients treated with cabazitaxel according to PSA and absolute monocyte count, and visceral metastasis stratified according to three risk groups $(\mathrm{P}<0.001)$. reported that PSA does not necessarily follow the exacerbation of the disease in the metastatic stage, suggesting that relying only on PSA changes in MCRPC patients may be misleading to clinicians with respect to making critical decisions with regard to whether to continue or change the current treatment $(15,16)$. However, in this study, univariate and multivariate analysis revealed PSA $\geq 100 \mathrm{ng} / \mathrm{ml}$ prior to cabazitaxel treatment $(\mathrm{P}=0.002)$ was significantly associated with shorter OS, suggesting the importance of PSA and the earlier introduction of cabazitaxel (Table II). Regarding the prognostic factors for PFS or OS in MCRPC patients treated with cabazitaxel, a recent paper reported the significance of anemia, low albumin, alkaline phosphatase level, the burden of bone metastasis or quantitative evaluation by automated bone scan index (17-19). A post hoc analysis of the TROPIC trial and a retrospective analysis have suggested that the occurrence of severe neutropenia with cabazitaxel was associated with a prolonged OS $(20,21)$.

There is a growing body of evidence suggesting that an interactive relationship between hemostatic factors and 
tumor biology might play a pivotal role in tumor development and progression. Monocytes are known to be able to alter the tumor microenvironment when recruited to tumor sites. An elevated absolute monocyte count was reported to be an adverse prognostic factor for hematological malignant diseases. Previous studies suggested that tumor-associated macrophages detected in tumor sites were described as a differentiated form of circulating monocytes outside the vasculature; a monocyte-macrophage lineage. The association of peripheral monocyte count and prostate cancer progression is not well characterized. Our recent study in CRPC patients receiving docetaxel therapy revealed that the elevated absolute monocyte count was associated with shorter OS (22). Of note, in this study, univariate and multivariate analysis revealed reduced absolute monocyte count $(\mathrm{P}=0.002)$ were significantly associated with shorter OS $(\mathrm{HR}=2.955 ; 95 \%$ CI 1.219-7.165, $\mathrm{P}=0.026$ ), suggesting an inverse immune microenvironment between the patients treated with docetaxel and cabazitaxel. As shown in Fig. 2, significant differences in OS were shown by stratifying the cohort study by the number of risk factors. To the best of our knowledge, this is the first study to identify the prognostic value of reduced absolute monocyte count in patients with $\mathrm{mCRPC}$ receiving cabazitaxel.

The strength of study was the indicated independence of absolute monocyte count from other prognostic factors, such as visceral metastasis or high PSA value. In addition, these prognostic factors can be easily obtained from laboratory test results and imaging studies, which are conducted routinely before the assessment of mCRPC with no additional financial burden. Therefore, adopting monocyte count evaluation into practice may provide additional information for clinical decision-making.

We acknowledge there were several limitations in our study. The study design was retrospective and involved a relatively small population. Therefore, the conclusions of this retrospective study need to be further explored and validated. A prospective study based on our study results is warranted to prove whether or not these predictive factors could be useful in a Japanese clinical setting.

In conclusion, we identified reduced monocyte count, as well as PSA $\geq 100 \mathrm{ng} / \mathrm{ml}$ and visceral metastasis, as potential predictors of worse prognosis during cabazitaxel therapy in Japanese mCRPC patients. The practical implications of our results may lead to tailoring of cabazitaxel introduction.

\section{Acknowledgements}

Not applicable.

\section{Funding}

The present study was supported in part by a Grant-in-Aid for Scientific Research (grant no. 17K11158 to Takeo Kosaka) from the Ministry of Education, Culture, Sports, Science, and Technology of Japan.

\section{Availability of data and materials}

The datasets generated and/or analyzed during the current study are not publicly available due to the lack of ethics approval for the purpose, but are available from the corresponding author on reasonable request.

\section{Authors' contributions}

TK contributed to the conception, design, acquisition, analysis and interpretation of data, and drafting of the manuscript. $\mathrm{HH}$, $\mathrm{RM}$ and MO took part in acquisition and analysis of data. MO took part in interpretation of data and drafting of the manuscript. All the authors have read and approved the final version of this manuscript.

\section{Ethics approval and consent to participate}

This study complied with the standards of the Declaration of Helsinki and the current ethical guidelines, and was approved by the Institutional Ethics Board of Keio University Hospital, which exempted obtaining informed consent because our study design was retrospective and all identifying information was removed.

\section{Patient consent for publication}

Not applicable.

\section{Competing interests}

The authors declare that they have no conflict of interest for this study.

\section{References}

1. Negoita S, Feuer EJ, Mariotto A, Cronin KA, Petkov VI, Hussey SK, Benard V, Henley SJ, Anderson RN, Fedewa S, et al: Annual Report to the Nation on the Status of Cancer, part II: Recent changes in prostate cancer trends and disease characteristics. Cancer 124: 2801-2814, 2018.

2. Cronin KA, Lake AJ, Scott S, Sherman RL, Noone AM, Howlader N, Henley SJ, Anderson RN, Firth AU, Ma J, et al: Annual Report to the Nation on the Status of Cancer, part I: National cancer statistics. Cancer 124: 2785-2800, 2018.

3. Siegel RL, Miller KD and Jemal A: Cancer statistics, 2018. CA Cancer J Clin 68: 7-30, 2018.

4. Siegel RL, Miller KD and Jemal A: Cancer statistics, 2017. CA Cancer J Clin 67: 7-30, 2017.

5. Akaza H, Onozawa $M$ and Hinotsu S: Prostate cancer trends in Asia. World J Urol 35: 859-865, 2017.

6. de Bono JS, Oudard S, Ozguroglu M, Hansen S, Machiels JP, Kocak I, Gravis G, Bodrogi I, Mackenzie MJ, Shen L, et al: Prednisone plus cabazitaxel or mitoxantrone for metastatic castration-resistant prostate cancer progressing after docetaxel treatment: A randomised open-label trial. Lancet 376: 1147-1154, 2010.

7. Mita AC, Denis LJ, Rowinsky EK, Debono JS, Goetz AD, Ochoa L, Forouzesh B, Beeram M, Patnaik A, Molpus K, et al: Phase I and pharmacokinetic study of XRP6258 (RPR 116258A), a novel taxane, administered as a 1-hour infusion every 3 weeks in patients with advanced solid tumors. Clin Cancer Res 15: 723-730, 2009.

8. Pivot X, Koralewski P, Hidalgo JL, Chan A, Gonçalves A, Schwartsmann G, Assadourian S and Lotz JP: A multicenter phase II study of XRP6258 administered as a 1-h i.v. infusion every 3 weeks in taxane-resistant metastatic breast cancer patients. Ann Oncol 19: 1547-1552, 2008.

9. Pezaro CJ, Omlin AG, Altavilla A, Lorente D, Ferraldeschi R, Bianchini D, Dearnaley D, Parker C, de Bono JS and Attard G: Activity of cabazitaxel in castration-resistant prostate cancer progressing after docetaxel and next-generation endocrine agents. Eur Urol 66: 459-465, 2014. 
10. Al Nakouzi N, Le Moulec S, Albigès L, Wang C, Beuzeboc P, Gross-Goupil M, de La Motte Rouge T, Guillot A, Gajda D, Massard C, et al: Cabazitaxel remains active in patients progressing after docetaxel followed by novel androgen receptor pathway targeted therapies. Eur Urol 68: 228-235, 2015.

11. Kosaka T and Oya M: Hemorrhagic cystitis in a patient without a past history of radiation therapy who was treated with cabazitaxel for CRPC. Ann Oncol 26: 2355-2356, 2015.

12. Watanabe K, Kosaka T, Hongo H, Tamaki S and Oya M: Headache caused by brain metastases of castration-resistant prostate cancer during cabazitaxel therapy. Keio J Med 66: 65-71, 2017.

13. Mukai H, Takahashi S, Nozawa M, Onozawa Y, Miyazaki J, Ohno K and Suzuki K; TED 11576 investigators: Phase 1 dose-escalation and pharmacokinetic study (TED 11576) of cabazitaxel in Japanese patients with castration-resistant prostate cancer. Cancer Chemother Pharmacol 73: 703-710, 2014.

14. Nozawa M, Mukai H, Takahashi S, Uemura H, Kosaka T, Onozawa Y, Miyazaki J, Suzuki K, Okihara K, Arai Y, et al: Japanese phase I study of cabazitaxel in metastatic castration-resistant prostate cancer. Int J Clin Oncol 20 1026-1034, 2015.

15. Armstrong AJ, Garrett-Mayer E, Ou Yang YC, Carducci MA, Tannock I, de Wit R and Eisenberger M: Prostate-specific antigen and pain surrogacy analysis in metastatic hormone-refractory prostate cancer. J Clin Oncol 25: 3965-3970, 2007.

16. Armstrong AJ, Garrett-Mayer E, de Wit R, Tannock I and Eisenberger M: Prediction of survival following first-line chemotherapy in men with castration-resistant metastatic prostate cancer. Clin Cancer Res 16: 203-211, 2010.
17. Bando Y, Hinata N, Terakawa T, Furukawa J, Harada KI, Nakano Y and Fujisawa M: Activity of cabazitaxel in patients with metastatic castration-resistant prostate cancer after treatment with single or dual regimens of novel androgen receptor-targeting agents. Med Oncol 34: 163, 2017.

18. Belderbos BPS, de Wit R, Hoop EO, Nieuweboer A, Hamberg P, van Alphen RJ, Bergman A, van der Meer N, Bins S, Mathijssen RHJ and van Soest RJ: Prognostic factors in men with metastatic castration-resistant prostate cancer treated with cabazitaxel. Oncotarget 8: 106468-106474, 2017.

19. Uemura K, Miyoshi Y, Kawahara T, Ryosuke J, Yamashita D, Yoneyama S, Yokomizo Y, Kobayashi K, Kishida T, Yao M and Uemura H: Prognostic value of an automated bone scan index for men with metastatic castration-resistant prostate cancer treated with cabazitaxel. BMC Cancer 18: 501, 2018.

20. Meisel A, von Felten S, Vogt DR, Liewen H, de Wit R, de Bono J, Sartor O and Stenner-Liewen F: Severe neutropenia during cabazitaxel treatment is associated with survival benefit in men with metastatic castration-resistant prostate cancer (mCRPC): A post-hoc analysis of the TROPIC phase III trial. Eur J Cancer 56: 93-100, 2016.

21. Kosaka T, Shinojima T, Morita S and Oya M: Prognostic significance of grade 3/4 neutropenia in Japanese prostate cancer patients treated with cabazitaxel. Cancer Sci 109: 1570-1575, 2018.

22. Shigeta K, Kosaka T, Kitano S, Yasumizu Y, Miyazaki Y, Mizuno R, Shinojima T, Kikuchi E, Miyajima A, Tanoguchi $\mathrm{H}$, et al: High absolute monocyte count predicts poor clinical outcome in patients with castration-resistant prostate cancer treated with docetaxel chemotherapy. Ann Surg Oncol 23: 4115-4122, 2016 